Article

\title{
Single-Beam Acoustic Tweezer Prepared by Lead-Free KNN-Based Textured Ceramics
}

\author{
Yi Quan ${ }^{1,2, *}$, Chunlong Fei ${ }^{1, *}$, Wei Ren ${ }^{2, *}$, Lingyan Wang ${ }^{2}$, Jinyan Zhao ${ }^{2}$, Jian Zhuang ${ }^{2}$, Tianlong Zhao ${ }^{1}$, \\ Zhaoxi Li ${ }^{1}$, Chenxi Zheng ${ }^{1}$, Xinhao Sun ${ }^{1}$, Kun Zheng ${ }^{2}$, Zhe Wang ${ }^{2}$, Matthew Xinhu Ren ${ }^{3}$, Gang Niu ${ }^{2}$, \\ Nan Zhang ${ }^{2}$, Tomoaki Karaki ${ }^{4}$, Zhishui Jiang ${ }^{5}$ and Li Wen ${ }^{5}$
}

check for

updates

Citation: Quan, Y.; Fei, C.; Ren, W.; Wang, L.; Zhao, J.; Zhuang, J.; Zhao, T.; Li, Z.; Zheng, C.; Sun, X.; et al. Single-Beam Acoustic Tweezer Prepared by Lead-Free KNN-Based Textured Ceramics. Micromachines 2022, 13, 175. https://doi.org/ $10.3390 / \mathrm{mi} 13020175$

Academic Editor: Klaus Stefan Drese

Received: 28 December 2021

Accepted: 24 January 2022

Published: 25 January 2022

Publisher's Note: MDPI stays neutral with regard to jurisdictional claims in published maps and institutional affiliations.

Copyright: (C) 2022 by the authors. Licensee MDPI, Basel, Switzerland. This article is an open access article distributed under the terms and conditions of the Creative Commons Attribution (CC BY) license (https:// creativecommons.org/licenses/by/ $4.0 /)$.
1 School of Microelectronics, Xidian University, Xi'an 710071, China; zhaot1@xidian.edu.cn (T.Z.); lizhaoxivip@163.com (Z.L.); xidianzcx@163.com (C.Z.); xinhaosun@126.com (X.S.)

2 Electronic Materials Research Laboratory, Key Laboratory of the Ministry of Education \& International Center for Dielectric Research, School of Electronic Science and Engineering, Xi'an Jiaotong University, Xi'an 710049, China; 1.y.wang@mail.xjtu.edu.cn (L.W.); zhaojy7@xjtu.edu.cn (J.Z.); jzhuang@xjtu.edu.cn (J.Z.); kkxxhhppyydd@163.com (K.Z.); wzhe1013@163.com (Z.W.); gangniu@xjtu.edu.cn (G.N.); nzhang1@xjtu.edu.cn (N.Z.)

3 Biology Program, Faculty of Science, The University of British Columbia, Vancouver, BC V6T 1Z4, Canada; xinhuren2018@gmail.com

4 Department of Intelligent Systems Design Engineering, Faculty of Engineering, Toyama Prefectural University, 5180 Kurokawa, Imizu 939-0398, Toyama, Japan; chen@pu-toyama.ac.jp

5 Guangdong JC Technological Innovation Electronics Co., Ltd., Zhaoqing 526000, China; loong_jzs@163.com (Z.J.); wlapmz@163.com (L.W.)

* Correspondence: yiquan-xjtu@foxmail.com (Y.Q.); clfei@xidian.edu.cn (C.F.); wren@mail.xjtu.edu.cn (W.R.)

\begin{abstract}
Acoustic tweezers for microparticle non-contact manipulation have attracted attention in the biomedical engineering field. The key components of acoustic tweezers are piezoelectric materials, which convert electrical energy to mechanical energy. The most widely used piezoelectric materials are lead-based materials. Because of the requirement of environmental protection, lead-free piezoelectric materials have been widely researched in past years. In our previous work, textured lead-free $(\mathrm{K}, \mathrm{Na}) \mathrm{NbO}_{3}(\mathrm{KNN})$-based piezoelectric ceramics with high piezoelectric performance were prepared. In addition, the acoustic impedance of the KNN-based ceramics is lower than that of lead-based materials. The low acoustic impedance could improve the transmission efficiency of the mechanical energy between acoustic tweezers and water. In this work, acoustic tweezers were prepared to fill the gap between lead-free piezoelectric materials research and applications. The tweezers achieved $13 \mathrm{MHz}$ center frequency and $89 \%-6 \mathrm{~dB}$ bandwidth. The $-6 \mathrm{~dB}$ lateral and axial resolution of the tweezers were $195 \mu \mathrm{m}$ and $114 \mu \mathrm{m}$, respectively. Furthermore, the map of acoustic pressure measurement and acoustic radiation calculation for the tweezers supported the trapping behavior for $100 \mu \mathrm{m}$ diameter polystyrene microspheres. Moreover, the trapping and manipulation of the microspheres was achieved. These results suggest that the KNN-based acoustic tweezers have a great potential for further applications.
\end{abstract}

Keywords: acoustic tweezer; non-contact manipulation; ultrasound; lead-free; piezoelectric; textured ceramics

\section{Introduction}

Non-contact manipulation for microparticles has attracted attention in the biomedical engineering field [1,2]. As of now, the most widely employed methods for non-contact manipulate microspheres and cells are optical tweezers [3,4]. However, there are several limitations for the use of optical tweezers. First of all, the optical tweezer can only be applied on optically transparent objects [1,5]. Secondly, the trapping force is weak (at piconewton range) and the optical tweezer can only handle small objectives at a level of several micrometers or nanometers $[5,6]$. Finally, for bio-samples such as cells or bacteria, 
the high energy generated by the focused light beam may damage the bio-samples [6]. An acoustic tweezer is a method that can spatially manipulate micro-particles and cells without contact [1]. The trapping force of an acoustic tweezer is significantly higher than an optical tweezer. For bio-samples, sound is much safer than light. In addition, the trapping range of an acoustic tweezer is much larger than an optical tweezer [5,7].

The most important component of acoustic tweezers is piezoelectric material [8]. Piezoelectric materials enable the conversion of electrical energy to mechanical energy [9-11]. The most widely used piezoelectric materials are $\mathrm{Pb}(\mathrm{Zr}, \mathrm{Ti}) \mathrm{O}_{3}(\mathrm{PZT})$-based ceramics with high piezoelectric properties [12,13]. To date, most acoustic tweezers have been based on LN single crystals or PZT-based ceramics. A single-beam acoustic tweezer has been utilized to trap microspheres and cells based on $193 \mathrm{MHz}$ which was prepared using $\mathrm{LiNbO}_{3}(\mathrm{LN})$ single crystals [5]. Moreover, $30 \mathrm{MHz}$ acoustic tweezers by LN single crystals were used to trap cancer cells and quantify mechanical properties without any contact [6]. Zhu et al. reported $50 \mathrm{MHz}$ single-beam acoustic tweezers that were prepared by PZT-based thick films [8].

However, there are several limitations to LN single crystals or PZT-based ceramics used on acoustic tweezers. The most significant restriction for PZT-based ceramics is lead which is harmful to the environment and human health [14-17]. Instructions have been issued to limit the use of lead in industry [18,19]. Meanwhile, LN single crystals are not high-performance piezoelectric materials with about $49 \mathrm{pC} / \mathrm{N} d_{33}$ value [20]. To replace the PZT-based ceramics and LN single crystals, lead-free piezoelectric materials have been well researched. For example, $(\mathrm{K}, \mathrm{Na}) \mathrm{NbO}_{3}(\mathrm{KNN})$-based piezoelectric ceramics are one of the most promising candidate. Saito et al. reported high-performance textured KNNbased lead-free ceramics prepared by reactive templated grain growth (RTGG) method [14]. Recently, several great works for KNN-based ceramics have been reported. $\mathrm{Xu}$ et al. reported a $570 \mathrm{pC} / \mathrm{N} d_{33}$ value in non-textured $\mathrm{KNN}$-based ceramics [21], and Li et al. achieved superior piezoelectric properties $\left(d_{33} \approx 700 \mathrm{pC} / \mathrm{N}\right.$ and $\left.d_{33} * 980 \mathrm{pm} / \mathrm{V}\right)$ at KNN-based textured ceramics [18].

Besides the limitations of lead and piezoelectric properties, the high acoustic impedance of both PZT-based ceramics and LN single crystals also restrict their usage in the acoustic tweezer field [7]. As we know, the transparency of sound waves is dependent on the acoustic impedance ratio between the piezoelectric materials and water [7,22]. Because of the mismatch of acoustic impedance, acoustic energy transferred between mediums is reflected. Thus, very little energy can transfer into the water and the trapping force would be low [7]. The acoustic impedances of KNN-based ceramics are much lower than those of PZT-based ceramics and LN single crystals which would greatly improve the acoustic energy transparency between the piezoelectric materials and water.

In our previous work, textured $0.915\left(\mathrm{~K}_{0.45} \mathrm{Na}_{0.5} \mathrm{Li}_{0.05}\right) \mathrm{NbO}_{3}-0.075 \mathrm{BaZrO}_{3}-0.01\left(\mathrm{Bi}_{0.5} \mathrm{Na}_{0.5}\right) \mathrm{TiO}_{3}$ (KNLN-BZ-BNT) ceramics with high piezoelectric properties, superior thermal stability and good fatigue resistance were prepared $[23,24]$. In this paper, we have prepared acoustic tweezers using textured KNN-based ceramics. The advantages of the textured KNN-based ceramics for acoustic tweezers are illustrated and compared to LN and PZT-based ceramics. Besides, the pulse-echo, impedance, insertion-loss, and resolution of the tweezers have been measured. Furthermore, the acoustic pressure field of the tweezers was simulated by finite elements analysis software and measured by hydrophone. The acoustic radiation of microspheres in the acoustic pressure field was calculated. Finally, the $100 \mu \mathrm{m}$ diameter microspheres were trapped and manipulated by the KNN-based acoustic tweezers.

\section{Materials and Methods}

The preparation and measurement of the KNN-based textured ceramics were illustrated in our previous manuscript. In the simulation of the acoustic pressure field, the finite elements analysis software COMSOL was used. Besides, the theoretical of acoustic radiation force (ARF) calculation were illustrated in our previous work [25]. 
The properties of the KNN-based textured ceramics are shown in Table 1. As a comparison, properties of PZT-5H (most common used PZT type) ceramics and LN single crystals are shown in Table 1 too. The piezoelectric response $d_{33}$ of KNN-based textured ceramics is $319 \mathrm{pC} / \mathrm{N}$, which are slightly lower than $585 \mathrm{pC} / \mathrm{N}$ for PZT-5H ceramics and much higher than $49 \mathrm{pC} / \mathrm{N}$ for LN single crystals. The dielectric constant $\left(\varepsilon_{\mathrm{r}}\right)$ of the KNNbased textured ceramics is 1651, which is the lower half of PZT-5H ceramics. Furthermore, the $\varepsilon_{\mathrm{r}}$ for LN single crystals is only 39 . In addition, the acoustic impedance $\left(Z_{\mathrm{a}}\right)$ of PZT-5H ceramics and LN single crystals are both about 35.5 MRayl which is much higher than the $Z_{\mathrm{a}}$ value 25.5 MRayl of KNN-based textured ceramics.

Table 1. Properties of piezoelectric materials.

\begin{tabular}{cccccccc}
\hline & $\rho\left(\mathbf{k g} / \mathbf{m}^{\mathbf{3}}\right)$ & $d_{\mathbf{3 3}}(\mathbf{p C} / \mathbf{N})$ & $k_{\mathbf{t}}$ & $\varepsilon_{\mathbf{r}}$ & $\tan \delta$ & $c(\mathbf{m} / \mathbf{s})$ & $Z_{\mathbf{a}}(\mathbf{M R a y l})$ \\
\hline KNN KNLN-BZ-BNT & 4500 & 319 & 0.42 & 1651 & 0.035 & 5610 & 25.2 \\
LN [22] & 4700 & 49 & 0.49 & 39 & 0.001 & 7360 & 34.5 \\
PZT-5H [26] & 7500 & 585 & 0.51 & 3400 & 0.02 & 4580 & 34.4 \\
\hline
\end{tabular}

In the preparation of the acoustic tweezers, the KNN-based ceramics were polished into $180 \mu \mathrm{m}$ in thickness. The $\mathrm{Cr} / \mathrm{Au}(50 \mathrm{~nm} / 100 \mathrm{~nm})$ electrodes were sputtered on both sides of the ceramics. Ag-epoxy were then put on the ceramics and polished into $20 \mu \mathrm{m}$ as matching layer. Next, the ceramics-matching layer was cut into squares with $3.5 \mathrm{~mm}$ length of side. Then an E-solder 3022 backing layer was placed on the backing side. Then, the elements were cut into a cylinder with a $3 \mathrm{~mm}$ diameter and a $2 \mathrm{~mm}$ thickness. The elements were fixed in copper housing and connected to SMA connectors. Following this, the acoustic tweezers were pressed by a steel ball with $4 \mathrm{~mm}$ diameter. Finally, a $2 \mu \mathrm{m}$-thick parylene $C$ layer was deposited onto the acoustic tweezers as the protective layer and covering layer by a Labcoator (PDS 2010, Specialty Coating Systems, Indianapolis, IN, USA). A picture of the acoustic tweezers is shown in Figure 1a. The picture of the focused piezoelectric elements can be found in Figure 1b.

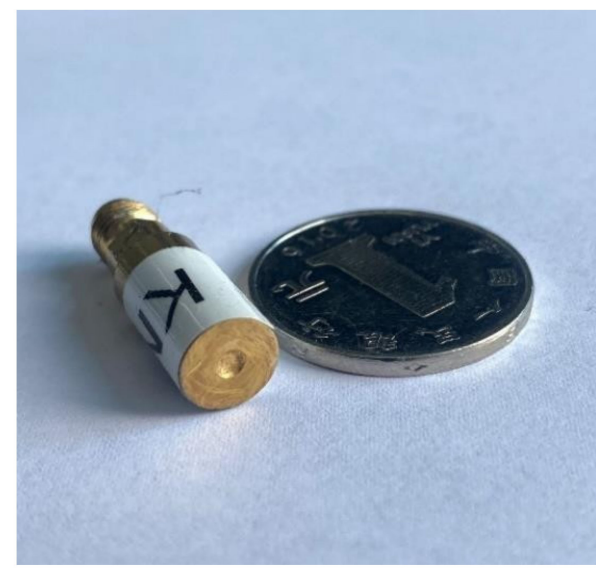

(a)

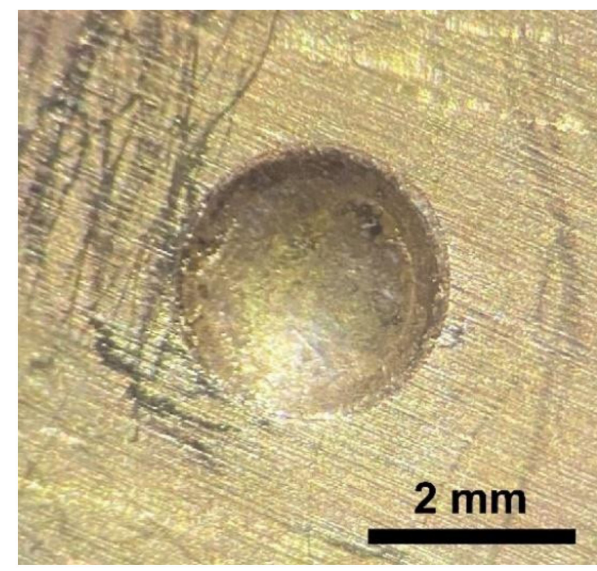

(b)

Figure 1. Pictures of (a) acoustic tweezer and (b) its piezoelectric element.

The pulse-echo measurement was tested under distilled water, by pulser/receiver (5073PR, Olympus, Bethlehem, PA, USA) with an electrical impulse at a $200 \mathrm{~Hz}$ repetition rate and $50 \Omega$-damping. The insertion loss was observed by a function generator (AFG3252C, Tektronix, Beaverton, OR, USA) and oscilloscope (TDS 5052, Tektronix). The resolution of the acoustic tweezers was evaluated by pulser/receiver (JSR Ultrasonics DPR 500, Imaginant, Pittsford, NY, USA) scanning three tungsten wire targets with $35 \mu \mathrm{m}$ diameter and a pig eye. The acoustic pressure field was measured by a hydrophone (NH1000, UK). 
The system of acoustic tweezer experiments is shown in Figure 2. The acoustic tweezers were fixed on a self-made fixture. The fixture was set on a three-axis motorized linear stage that controlled the movement of the acoustic tweezers. Meanwhile, a function generator (AFG3252C, Tektronix) and a $50 \mathrm{~dB}$ power amplifier (525 LA, ENI Rochester, Rochester, NY, USA) were used to drive the acoustic tweezers. Photographs and movies of the trapped motions of the microsphere were taken by a microscope (LIOO, Beijing, China) with a CMOS camera. We used $100 \mu \mathrm{m}$ polystyrene (PS) microspheres (Ruige, Luoyang, China) as the trapping targets.

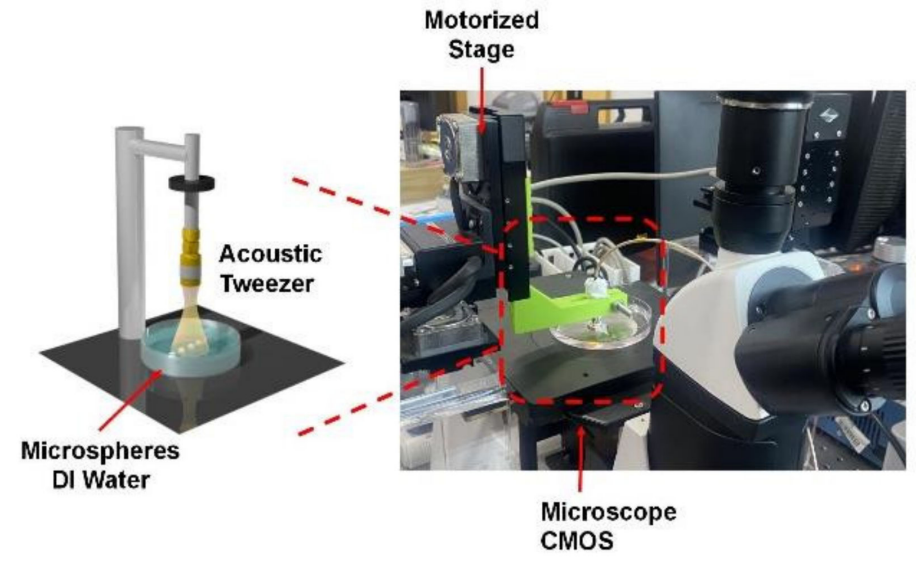

Figure 2. The diagram of acoustic tweezer system.

\section{Results}

The electrical impedance and pulse-echo response are shown in Figure 3. The electrical impedance can be found in Figure 3a. As with the simulated data, there were two resonance peaks at $8 \mathrm{MHz}$ and $16.8 \mathrm{MHz}$. The peak at $8 \mathrm{MHz}$ was much higher than the peak at $16.8 \mathrm{MHz}$. In addition, the impedance of acoustic tweezers at $13 \mathrm{MHz}$ was near $70 \Omega$, which is near the electrical matching of $50 \Omega$. The pulse-echo measurement illustrated the send and receive performance of the acoustic tweezers, which is shown in Figure $3 \mathrm{~b}$. The center frequency was $13 \mathrm{MHz}$, and the $-6 \mathrm{~dB}$ bandwidth was $89 \%$. Meanwhile, the peak-to-peak voltage was about $600 \mathrm{mV}$. Because of the high sensitivity and board $-6 \mathrm{~dB}$ bandwidth, the KNN-based acoustic tweezers can be excited under a large range of frequency.

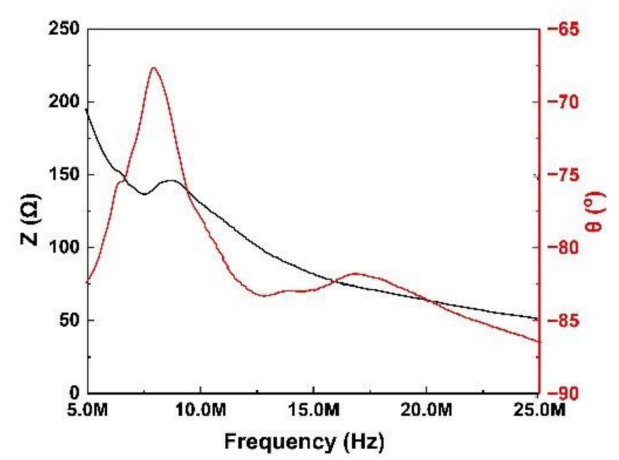

(a)

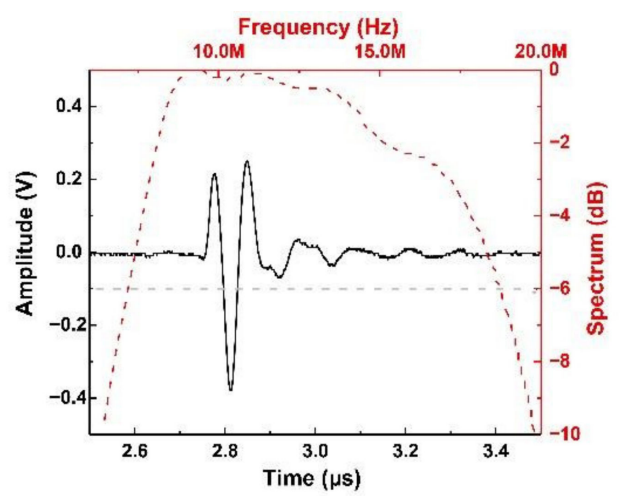

(b)

Figure 3. (a) Electrical magnitude (black) and phase angle (red), (b) pulse-echo wave (black) and frequency spectrum (red) performances of acoustic tweezers by textured KNN-based ceramics.

The value of insertion-loss could evaluate the availability of electromechanical efficiency of the acoustic tweezers. The two-way insertion-loss of the acoustic tweezers can be found in Figure 4. The optimal insertion-loss was found at $9 \mathrm{MHz}$ with $-29 \mathrm{~dB}$. 
Furthermore, the insertion-loss in $8 \mathrm{MHz}$ to $17 \mathrm{MHz}$ was retained higher than $-33 \mathrm{~dB}$, which means the acoustic tweezers could achieve high sensitivity under a wide excitation/ receiving frequency.

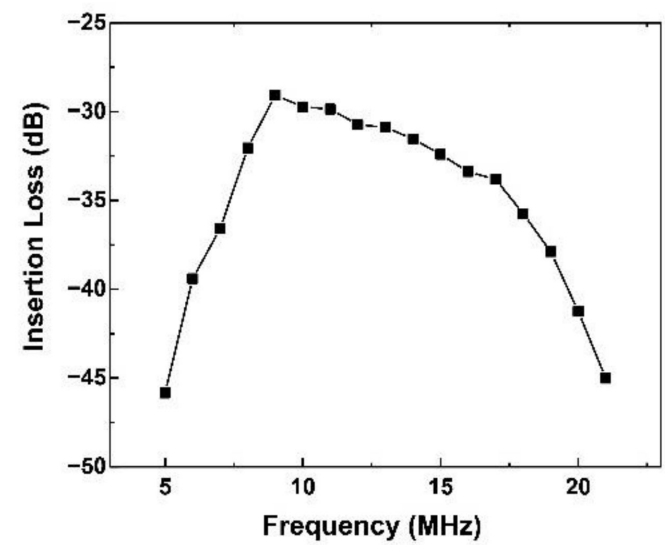

Figure 4. Two-way insertion-loss of KNN-based acoustic tweezers.

To determine whether the focused process was effective on the acoustic tweezers, four tungsten wire targets with a $35 \mu \mathrm{m}$ diameter were used to test the resolution of the acoustic tweezers. Figure 5 shows the phantom image. The measured $-6 \mathrm{~dB}$ lateral and axial resolutions estimated by Figure 5 are shown in Figure 6.

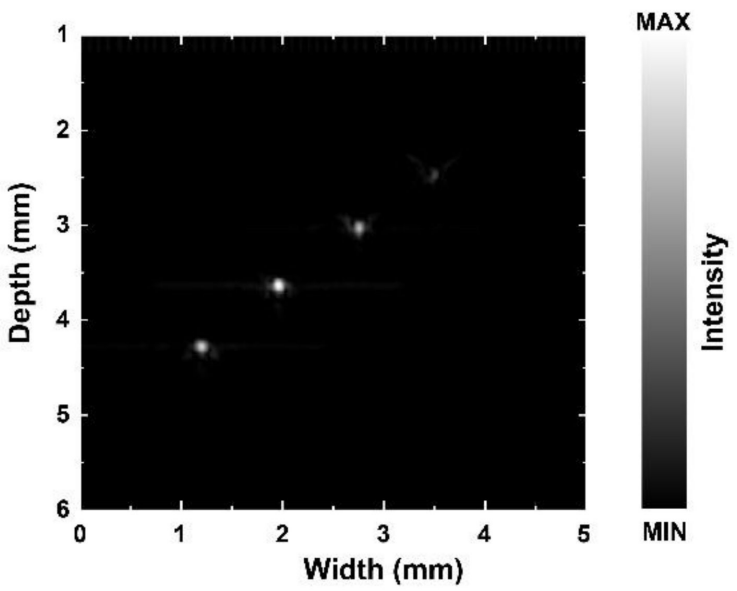

Figure 5. Tungsten wire targets image by KNN-based acoustic tweezers.

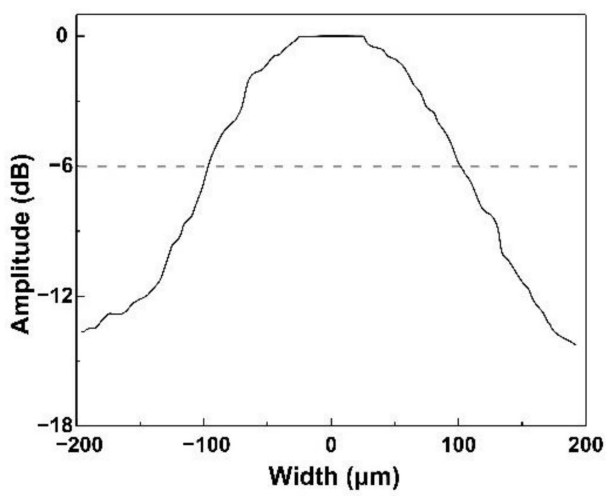

(a)

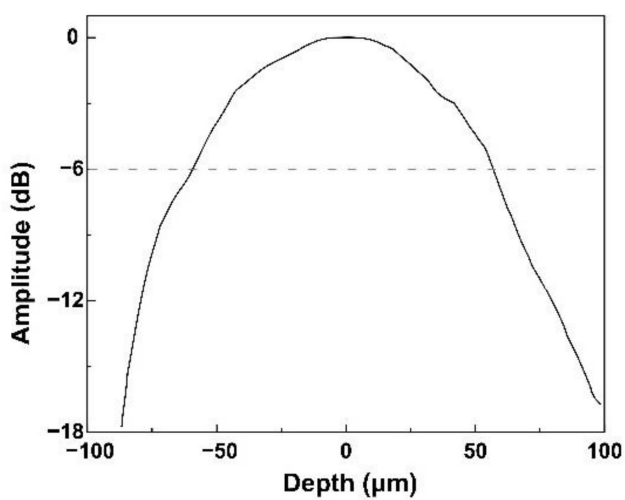

(b)

Figure 6. (a) Lateral and (b) axial resolution of KNN-based acoustic tweezers. 
The $-6 \mathrm{~dB}$ lateral resolution was $195 \mu \mathrm{m}$, and the $-6 \mathrm{~dB}$ axial resolution was $114 \mu \mathrm{m}$. As comparisons, the theoretical values of resolution have been calculated by the following formulas [7]:

$$
\begin{aligned}
& \text { Axial resolution } R_{a x}=\frac{\lambda}{2 \mathrm{BW}} \\
& \text { Lateral resolution } R_{\text {lat }}=f \lambda
\end{aligned}
$$

where $\mathrm{BW}$ is the $-6 \mathrm{~dB}$ bandwidth of the acoustic tweezers, $f$ is the $\mathrm{f}$-number (focal distance/diameter of piezoelectric element) of the acoustic tweezers, and $\lambda$ is the wavelength in water at the center frequency. The diameter of piezoelectric elements is $2 \mathrm{~mm}$, and the focal distance is also $2 \mathrm{~mm}$. Thus, the f-number of the acoustic tweezers is 1 . Based on the equations, the lateral resolution of the KNN-based acoustic tweezers is $119 \mu \mathrm{m}$ and the axial resolution is $62 \mu \mathrm{m}$. The measured lateral resolution is lower than the theoretical value, which might be due to the cracking during the focusing process. The excellent resolution shown that the acoustic tweezers were tightly focused.

The map of acoustic pressure has been simulated by a finite element method (FEM) in the COMSOL environment, which is shown in Figure 7a. The focal point of the acoustic tweezers was defined as zero point, and the piezo-elements were $2 \mathrm{~mm}$ away from the zero point. The high acoustic press area is about $2 \mathrm{~mm}$ length and $0.5 \mathrm{~mm}$ width. In addition, the measured acoustic pressure field is shown in Figure $7 \mathrm{~b}$. The distribution of the acoustic press field is similar to the simulation. There is a $2 \mathrm{~mm}$ length and $0.5 \mathrm{~mm}$ width high-intensity area. The results illustrates that the simulated map of acoustic pressure is highly reliable.

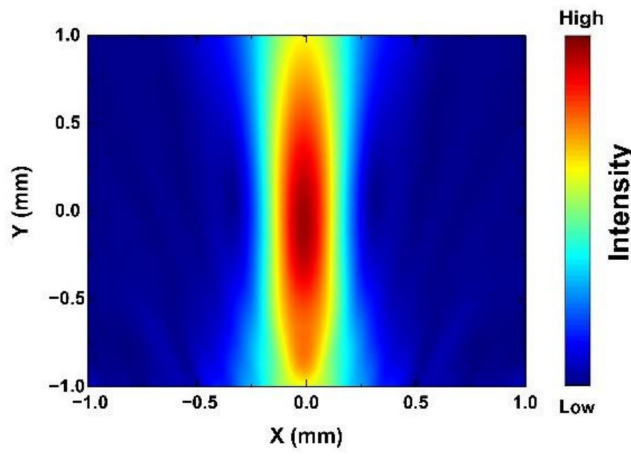

(a)

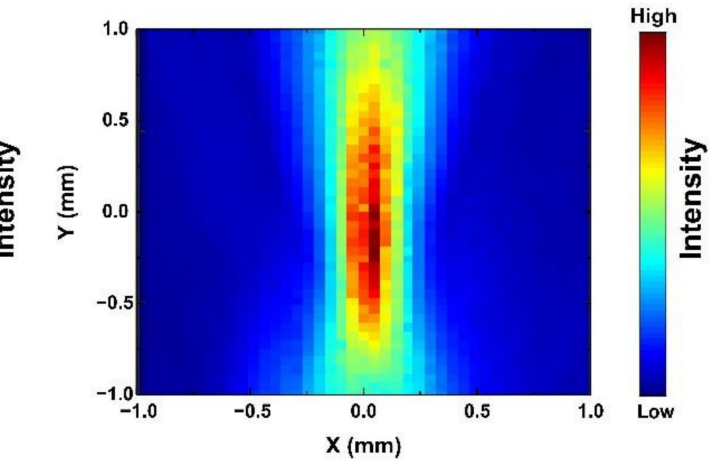

(b)

Figure 7. (a) Simulated and (b) measured map of acoustic pressure of KNN-based acoustic tweezers.

To further understand the trapping of microsphere, the acoustic radiation for $100 \mu \mathrm{m}$ diameter PS microspheres has been calculated by the FEM simulated acoustic press field. The acoustic radiation of the microsphere is the same in the $x$ and $y$ directions, and only the force with one dimension was calculated by the following formula $[25,27]$ :

$$
F_{x}=\frac{1}{8 \pi^{2} \rho c^{2} k^{2}} \operatorname{Re}\left\{\sum_{n=0}^{\infty} \Psi_{n} \sum_{m=-n}^{n} A_{m n}\left(H_{m n} H_{n+1, M+1}^{*}-H_{n,-m} H_{n+1,-m-1}^{*}\right)\right\}
$$

where the function

$$
H_{m n}=\iint_{k_{x}^{2}+k_{y}^{2} \leq k^{2}} d k_{x} d k_{y} S\left(k_{x}, k_{y}\right) Y_{n m}^{*}\left(\theta_{k}, \varphi_{k}\right)
$$

$k$ is the wavenumber in the fluid, $\theta_{k}$ is the spherical angle of the wave vector, $\varphi_{k}$ is the polar angles, $c$ is the sound velocity of the fluid, $Y_{n m}^{*}\left(\theta_{k}, \varphi_{k}\right)$ is spherical harmonics, $A_{m n}$ and $\Psi_{n}$ is

$$
A_{m n}=\sqrt{\frac{(n+m+1)(n+m+2)}{(2 n+1)(2 n+3)}}
$$




$$
\Psi_{n}=2\left(c_{n}+c_{n+1}^{*}+2 c_{n} c_{n}^{*}\right)
$$

respectively, where the * means the complex conjugation, $c_{n}$ is the scattering coefficients. The $S\left(k_{x}, k_{y}\right)$ is the angular spectrum of the acoustic wave generated by the acoustic tweezers. At its focal point:

$$
S\left(k_{x}, k_{y}\right)=\int_{-\infty}^{+\infty} \int_{-\infty} p(x, y) e^{-i k_{x} x-i k_{y} y} d x d y
$$

where $p(x, y)$ is the distribution of complex acoustic pressure generated by the acoustic tweezer. Base on those formulas, the acoustic radiation force can be calculated with knowledge of the propagation medium, the scatterer, and complex sound field distribution.

The lateral distributions of the lateral component of force $\left(F_{x}\right)$ on the xy plane for $100 \mu \mathrm{m}$ diameter PS microsphere are shown in Figure 8. In Figure 8a, the red areas indicate that the orientation of $F_{x}$ is the positive direction of the x-axis, and the blue areas means the $F_{x}$ forward to the negative direction of the x-axis. Plots for $F_{x}$ along the $\mathrm{y}=0$ line in the force map are shown in Figure $8 \mathrm{~b}$. When a microsphere moved to the left, the direction of $F_{x}$ was to the right. By contrast, when the microsphere moved to the right, the direction of $F_{x}$ was to the right. Based on this observation, it was concluded that the microparticles can be trapped by the KNN-based acoustic tweezers.

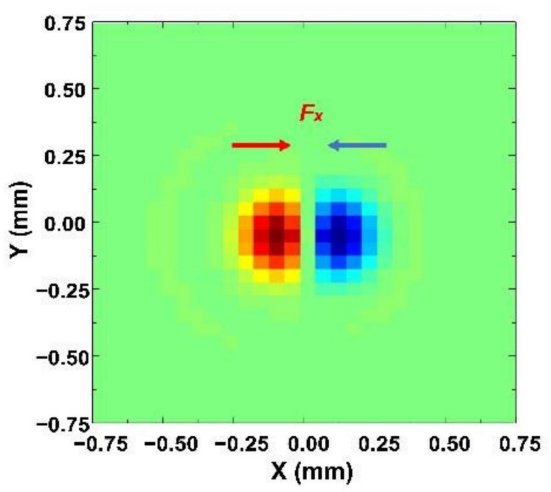

(a)

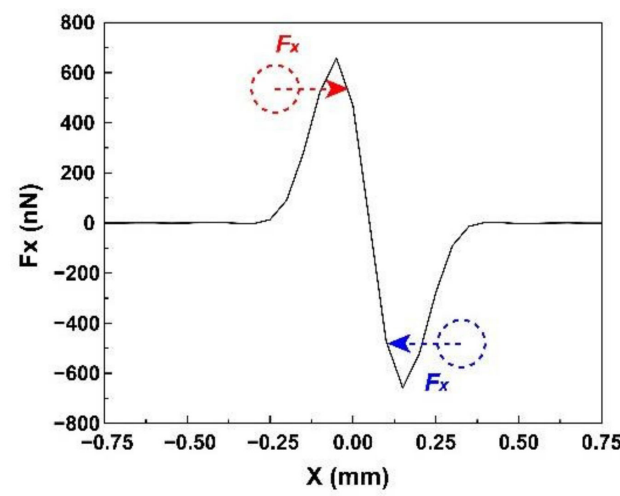

(b)

Figure 8. (a) Lateral distributions of the lateral component of force $\left(F_{x}\right)$ on the xy plane for $100 \mu \mathrm{m}$ diameter polystyrene (PS) microsphere. (b) Plots for acoustic radiation forces $F_{x}$ along the $\mathrm{y}=0$ line.

A single PS microsphere with a $100 \mu \mathrm{m}$ diameter was trapped and manipulated using the KNN-based acoustic tweezers, which is shown in Figure 9. The acoustic tweezers were driven by $10 \mathrm{MHz}$ excitation frequency, $10 \mathrm{mV}$ peak-to-peak voltage with $50 \mathrm{~dB}$ signal amplification, $5 \%$ duty cycle, and $1 \mathrm{kHz}$ pulse repetition frequency. As can be seen, a single microsphere was manipulated along the movement of the transducer. (The video can be found in Video S1) The arrows represent the movement direction of the transducer. The results demonstrate that the KNN-based textured ceramics could be used for acoustic tweezers. 


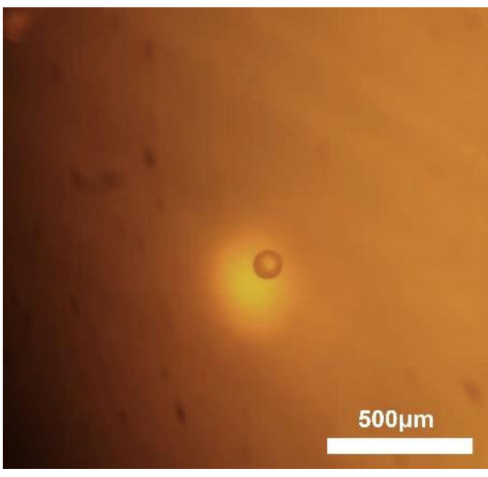

(a)

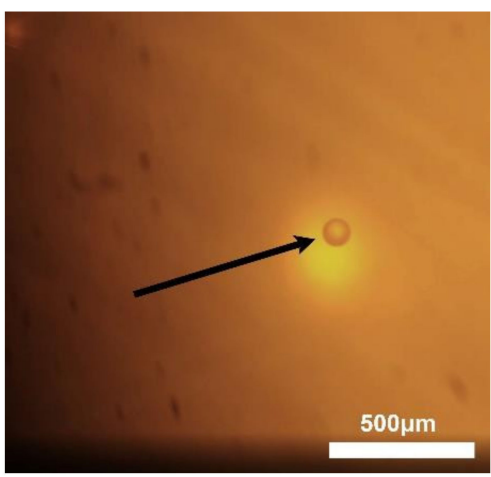

(c)

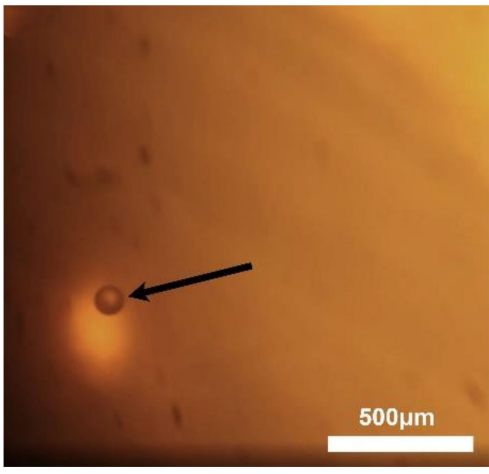

(b)

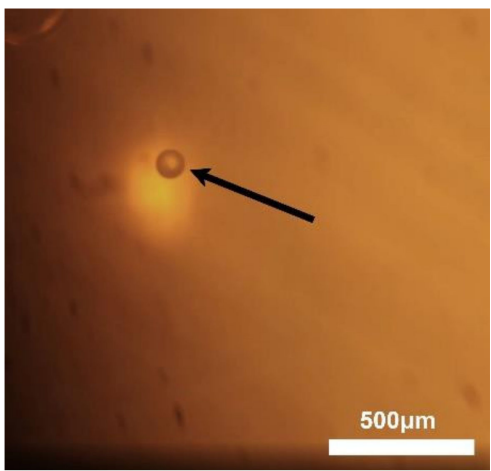

(d)

Figure 9. (a-d) show trapping and manipulation process of $100 \mu \mathrm{m}$ diameter PS microsphere by the KNN-based acoustic tweezers.

\section{Discussion}

In this work, novel lead-free ceramics such as $\mathrm{KNN}$-based, $(\mathrm{Bi}, \mathrm{Na}) \mathrm{TiO}_{3}$-based, $\mathrm{BiFeO}_{3}$ based ceramics, etc. were used in acoustic tweezers for the first time. In this field, there are several advantages to the textured KNLN-BZ-BNT ceramics besides environmental protection.

Compared to LN single crystals, the piezoelectric response of the textured $\mathrm{KNN}$ based ceramics is much higher $(319 \mathrm{pC} / \mathrm{N}$ to $49 \mathrm{pC} / \mathrm{N})$, which would greatly improve the acoustic radiation force to microspheres by $\mathrm{KNN}$-based acoustic tweezers. In addition, the machinability of ceramics is much better than that of single crystals. Moreover, the dielectric constant of the KNN-based ceramics is much lower than PZT-5H ceramics. The low dielectric constant would substantially help acoustic tweezers when the center frequency becomes high.

$Z_{\mathrm{a}}$ is one of the most important parameters for piezoelectric materials in acoustic applications as it determines the efficiency of mechanical energy transmission between different media. The transmission coefficients $(T)$ between piezoelectric materials and water with 0 of incident angle are as follows [7]:

$$
T=2 Z_{\mathrm{w}} /\left(Z_{\mathrm{w}}+Z_{\mathrm{a}}\right)
$$

where $Z_{\mathrm{a}}$ is the acoustic impendence of piezoelectric materials and $Z_{\mathrm{w}}$ is the acoustic impendence of water, which is about 1.5 MRayl. According to Equation (3), the closer the $Z_{\mathrm{a}}$ of piezoelectric materials to $1.5 \mathrm{MRayl}$, the higher the $T$ between piezoelectric materials and water. Most of the $Z_{a}$ of piezoelectric ceramics and single crystals are much higher than 1.5 MRayl. Thus, the lower $Z_{\mathrm{a}}$ would bring higher T. Among the properties of materials in Table 1, compared to PZT-5H ceramics and LN single crystals, the $Z_{\mathrm{a}}$ of KNN-based textured ceramics is much lower. The low $Z_{\mathrm{a}}$ could help the textured $\mathrm{KNN}$-based ceramics achieve a high mechanical energy transmission. For acoustic tweezer applications, the 
high mechanical energy transmission could greatly increase the acoustic radiation, finally enhancing the effect of microsphere trapping. By way of comparison, the pulse-echo measurements of PZT-based acoustic tweezers are shown in Figure S1. In addition, the trapping of the PS microsphere with a $20 \mu \mathrm{m}$ diameter by using KNN-based and PZT-based tweezers is shown in and Videos S2 and S3, respectively. Although the properties of KNNbased tweezers are slightly lower than the properties of the PZT-based tweezers, those results could illustrate that the novel lead-free ceramics have great potential on piezoelectric devices

\section{Conclusions}

Acoustic tweezers have been prepared by textured KNLN-BZ-BNT ceramics with $13 \mathrm{MHz}$ center frequency and $89 \%-6 \mathrm{~dB}$ bandwidth. The insertion-loss was $-29 \mathrm{~dB}$ at $9 \mathrm{MHz}$ and was retained higher than $-33 \mathrm{~dB}$ between $8 \mathrm{MHz}$ and $17 \mathrm{MHz}$. The $-6 \mathrm{~dB}$ lateral and axial resolutions of the acoustic tweezers were $195 \mu \mathrm{m}$ and $114 \mu \mathrm{m}$, respectively. The map of acoustic pressure was measured and the acoustic radiation for $100 \mu \mathrm{m}$ diameter PS microspheres was calculated to demonstrate the acoustic trapping. Then, $100 \mu \mathrm{m}$-diameter PS microspheres were trapped and multiplied by KNN-based acoustic tweezers. Furthermore, the acoustic impedance of the textured KNLN-BZ-BNT ceramics was 25.2 MRayl, which was much lower than most commonly used piezoelectric materials of PZT-5H and LN single crystals. Based on the results, the textured KNLN-BZ-BNT ceramics are a promising type of piezoelectric material for acoustic tweezer applications.

Supplementary Materials: The following supporting information can be downloaded at: https:/ / www.mdpi.com/article/10.3390/mi13020175/s1, Figure S1: Pulse-echo wave (black) and frequency spectrum (red) performances of acoustic tweezers by PZT-based ceramics. Video S1, Video S2, Video S3.

Author Contributions: Conceptualization, W.R. and C.F.; methodology, Y.Q., X.S. and C.Z.; supervision, W.R., L.W. (Lingyan Wang) and C.F.; formal analysis, Z.L.; investigation, K.Z. and Z.W.; writingoriginal draft preparation, Y.Q.; writing-review and editing, J.Z. (Jinyan Zhao), J.Z. (Jian Zhuang), L.W. (Lingyan Wang), G.N., N.Z. and M.X.R.; resources, T.K. and T.Z.; funding acquisition, Z.J. and L.W. (Li Wen). All authors have read and agreed to the published version of the manuscript.

Funding: This research was funded by the National Natural Science Foundation of China (Grants No. $51602243,51902246,51911530125,12174299)$, the China National Key R\&D Program (2020YFC0122100), Key Research Project of Shaanxi Province of China (Grant No. 2018ZDXM-GY-150), Xijiang Innovation Team Introduction Program of Zhaoqing, China Postdoctoral Science Foundation (Grants No. 2019M663697), the Natural Science Fundamental Research Project of Shaanxi Province of China (No. 2019JQ590), the "111 Project" of China (B14040) and the Fundamental Research Funds for the Central Universities, the Key Projects of National Defense Basic Research Program of National Defense Science and Industry Administration (No: JCKY2019210B003).

Data Availability Statement: Not applicable.

Conflicts of Interest: The authors declare no conflict of interest.

\section{References}

1. Ozcelik, A.; Rufo, J.; Guo, F.; Gu, Y.; Li, P.; Lata, J.; Huang, T.J. Acoustic tweezers for the life sciences. Nat. Methods 2018, 15, 1021-1028. [CrossRef] [PubMed]

2. $\quad$ Fei, C.; Li, Y.; Zhu, B.; Chiu, C.T.; Chen, Z.; Li, D.; Yang, Y.; Kirk Shung, K.; Zhou, Q. Contactless microparticle control via ultrahigh frequency needle type single beam acoustic tweezers. Appl. Phys. Lett. 2016, 109, 173509. [CrossRef] [PubMed]

3. Neuman, K.C.; Nagy, A. Single-molecule force spectroscopy: Optical tweezers, magnetic tweezers and atomic force microscopy. Nat. Methods 2008, 5, 491-505. [CrossRef] [PubMed]

4. $\quad$ Li, Z.; Yang, J.; Liu, S.; Jiang, X.; Wang, H.; Hu, X.; Xue, S.; He, S.; Xing, X. High throughput trapping and arrangement of biological cells using self-assembled optical tweezer. Opt. Express 2018, 26, 34665-34674. [CrossRef] [PubMed]

5. Lam, K.H.; Li, Y.; Li, Y.; Lim, H.G.; Zhou, Q.; Shung, K.K. Multifunctional single beam acoustic tweezer for non-invasive cell/organism manipulation and tissue imaging. Sci. Rep. 2016, 6, 37554. [CrossRef] [PubMed] 
6. Hwang, J.Y.; Kim, J.; Park, J.M.; Lee, C.; Jung, H.; Lee, J.; Shung, K.K. Cell deformation by single-beam acoustic trapping: A promising tool for measurements of cell mechanics. Sci. Rep. 2016, 6, 27238. [CrossRef]

7. Shung, K.K. Diagnostic Ultrasound: Imaging and Blood Flow Measurements; CRC press: Boca Raton, FL, USA, 2015.

8. Zhu, B.; Xu, J.; Li, Y.; Wang, T.; Xiong, K.; Lee, C.; Yang, X.; Shiiba, M.; Takeuchi, S.; Zhou, Q. Micro-particle manipulation by single beam acoustic tweezers based on hydrothermal PZT thick film. AIP Adv. 2016, 6, 035102. [CrossRef]

9. Uchino, K. Piezoelectric Actuators and Ultrasonic Motors; Springer Science \& Business Media: Norwell, MA, USA, 1996 ; Volume 1.

10. Zeng, Y.; Jiang, L.; Sun, Y.; Yang, Y.; Quan, Y.; Wei, S.; Lu, G.; Li, R.; Rong, J.; Chen, Y. 3D-Printing Piezoelectric Composite with Honeycomb Structure for Ultrasonic Devices. Micromachines 2020, 11, 713. [CrossRef]

11. Zheng, K.; Quan, Y.; Zhuang, J.; Zhao, J.; Ren, W.; Wang, L.; Wang, Z.; Niu, G.; Fei, C.; Jiang, Z. Achieving high piezoelectric performances with enhanced domain-wall contributions in <001>-textured Sm-modified PMN-29PT ceramics. J. Eur. Ceram. Soc. 2021, 41, 2458-2464. [CrossRef]

12. Zhao, J.; Zhang, N.; Quan, Y.; Niu, G.; Ren, W.; Wang, Z.; Zheng, K.; Zhao, Y.; Ye, Z.-G. Evolution of mesoscopic domain structure and macroscopic properties in lead-free $\mathrm{Bi}_{0.5} \mathrm{Na}_{0.5} \mathrm{TiO}_{-}-\mathrm{BaTiO}_{3}$ ferroelectric ceramics. J. Appl. Phys. 2021, 129, 084103. [CrossRef]

13. Jiang, L.; Xing, J.; Tan, Z.; Wu, J.; Chen, Q.; Xiao, D.; Zhu, J. High piezoelectricity in $(\mathrm{K}, \mathrm{Na})(\mathrm{Nb}, \mathrm{Sb}) \mathrm{O}_{3}-(\mathrm{Bi}, \mathrm{La}, \mathrm{Na}, \mathrm{Li}) \mathrm{ZrO}{ }_{3}$ lead-free ceramics. J. Mater. Sci. 2016, 51, 4963-4972. [CrossRef]

14. Saito, Y.; Takao, H.; Tani, T.; Nonoyama, T.; Takatori, K.; Homma, T.; Nagaya, T.; Nakamura, M. Lead-free piezoceramics. Nature 2004, 432, 84-87. [CrossRef] [PubMed]

15. Shrout, T.R.; Zhang, S.J. Lead-free piezoelectric ceramics: Alternatives for PZT? J. Electroceramics 2007, 19, 113-126. [CrossRef]

16. Zhang, S.; Xia, R.; Shrout, T.R. Lead-free piezoelectric ceramics vs. PZT? J. Electroceramics 2007, 19, 251-257. [CrossRef]

17. $\mathrm{Wu}$, J.; Xiao, D.; Zhu, J. Potassium-sodium niobate lead-free piezoelectric materials: Past, present, and future of phase boundaries. Chem. Rev. 2015, 115, 2559-2595. [CrossRef]

18. Li, P.; Zhai, J.; Shen, B.; Zhang, S.; Li, X.; Zhu, F.; Zhang, X. Ultrahigh piezoelectric properties in textured (K, Na)NbO- -based lead-free ceramics. Adv. Mater. 2018, 30, 1705171. [CrossRef]

19. Li, J.-F. Lead-Free Piezoelectric Materials; John Wiley \& Sons: Wollonggong, Australia, 2020; Chapter 3.

20. Chen, Z.; Zheng, L.; Cao, W.; Chen, X.; Chen, R.; Li, R.; Shung, K.; Zhou, Q. High-frequency ultrasonic imaging with lead-free $(\mathrm{Na}, \mathrm{K})(\mathrm{Nb}, \mathrm{Ta}) \mathrm{O}_{3}$ single crystal. Ultrason. Imaging 2017, 39, 348-356. [CrossRef]

21. Xu, K.; Li, J.; Lv, X.; Wu, J.; Zhang, X.; Xiao, D.; Zhu, J. Superior piezoelectric properties in potassium-sodium niobate lead-free ceramics. Adv. Mater. 2016, 28, 8519-8523. [CrossRef]

22. Chen, R.; Jiang, L.; Zhang, T.; Matsuoka, T.; Yamazaki, M.; Qian, X.; Lu, G.; Safari, A.; Zhu, J.; Shung, K.K. Eco-friendly highly sensitive transducers based on a new KNN-NTK-FM lead-free piezoelectric ceramic for high-frequency biomedical ultrasonic imaging applications. IEEE Trans. Biomed. Eng. 2018, 66, 1580-1587. [CrossRef]

23. Quan, Y.; Ren, W.; Niu, G.; Wang, L.; Zhao, J.; Zhang, N.; Liu, M.; Ye, Z.-G.; Liu, L.; Karaki, T. Large Piezoelectric strain with superior thermal stability and excellent fatigue resistance of lead-free potassium sodium niobate-based grain orientationcontrolled ceramics. ACS Appl. Mater. Interfaces 2018, 10, 10220-10226. [CrossRef]

24. Quan, Y.; Fei, C.; Ren, W.; Wang, L.; Niu, G.; Zhao, J.; Zhuang, J.; Zhang, J.; Zheng, K.; Lin, P. Lead-free KNN-based Textured Ceramics for High Frequency Ultrasonic Transducer Application. IEEE Trans. Ultrason. Ferroelectr. Freq. Control 2021, 68, 1979-1987. [CrossRef] [PubMed]

25. Li, Z.; Wang, D.; Fei, C.; Qiu, Z.; Hou, C.; Wu, R.; Li, D.; Zhang, Q.; Chen, D.; Chen, Z. The forbidden band and size selectivity of acoustic radiation force trapping. Iscience 2021, 24, 101988. [CrossRef] [PubMed]

26. Jaffe, H.; Berlincourt, D. Piezoelectric transducer materials. Proc. IEEE 1965, 53, 1372-1386. [CrossRef]

27. Sapozhnikov, O.A.; Bailey, M.R. Radiation force of an arbitrary acoustic beam on an elastic sphere in a fluid. J. Acoust. Soc. Am. 2013, 133, 661-676. [CrossRef] [PubMed] 